\title{
Serum 25-Hydroxyvitamin D Level and Extent and Complexity of Coronary Artery Disease
}

\author{
Taner Şeker, Mustafa Gür, Gülhan Yüksel Kalkan, Osman Kuloğlu, \\ Nermin Yıldız Koyunsever, Durmuş Yıldıray Şahin, ${ }^{*}$ Caner Türkoğlu, \\ Selahattin Akyol, Zafer Elbasan, Hazar Harbalıoğlu, and Murat Çaylı \\ Adana Numune Training and Research Hospital, Department of Cardiology, Adana, Turkey
}

\begin{abstract}
Background: There are limited number of studies about relationship between serum vitamin $\mathrm{D}$ level and presence and severity of coronary artery disease (CAD). We assessed the relationship between the extent and complexity of CAD assessed by SYNTAX score and 25-hydroxyvitamin D level in patients with stable CAD. Methods: In the study, 209 consecutive patients with stable CAD (age: $63.1 \pm 10.0$ years) and 102 healthy control subjects (age $61.3 \pm 13.7$ years) were included. Serum 25-hydroxyvitamin $D$ was measured using a direct competitive chemiluminescent immunoassay and other biochemical markers were measured in all subjects. All subjects underwent coronary angiography and

SS was calculated. Results: The mean serum 25-hydroxyvitamin $D$ level of CAD group was lower than the control group $(P<0,001)$. Multivariate regression analysis showed that serum 25-hydroxyvitamin $D$ level was independently associated with SYNTAX score $(\beta=-0.396, P<0.001)$, hypertension $(\beta=-0.183, P=0.003)$, high sensitive $\mathrm{C}$-reactive protein $(\beta=$ $-0.141, P=0.014)$, and body mass index $(\beta=-0.135, P=0.023)$ in patient group. Conclusion: 25 -Hydroxyvitamin D level was associated with extent and complexity of CAD. 25-Hydroxyvitamin D may play a role in pathogenesis and severity of coronary atherosclerosis. J. Clin. Lab. Anal. 28:5258, 2014. (c) 2013 Wiley Periodicals, Inc.

Key words: coronary; artery; vitamin D; SYNTAX; severity
\end{abstract}

\section{INTRODUCTION}

Serum 25-hydroxyvitamin D (vitamin D) deficiency or insufficiency is a substantially prevalent condition in patients with coronary artery disease (CAD) and in the general population $(1,2)$. Vitamin D deficiency is also associated with carotid atherosclerosis as well as coronary atherosclerosis (3). Vitamin D deficiency is associated with risk for cardiovascular event in the general population $(4,5)$. It is shown that there is a significant association between low vitamin D levels and an increased risk of CAD $(6,7)$. Moreover, vitamin D deficiency is independently associated with cardiovascular morbidity and mortality in the general population (8). In addition, it has recently been reported that vitamin D supplementation is significantly associated with better survival, specifically in patients with documented deficiency (9).

The synergy between percutaneous coronary intervention with taxus and cardiac surgery (SYNTAX) score quantifies extent and complexity of angiographic lesions
(10). It is able to aid revascularization decisions and predict long-term mortality and morbidity in patients with $\operatorname{CAD}(11,12)$.

Vitamin D plays a role in the pathogenesis of cardiovascular disease through a direct involvement in the process of plaque formation and progression $(8,13)$. Although low vitamin $\mathrm{D}$ level is associated with presence of CAD, relationship between vitamin $\mathrm{D}$ level and extent and complexity of CAD assessed with SYNTAX score has not been clearly determined yet. Therefore, we aimed to investigate the association between vitamin $\mathrm{D}$ level and the extent and complexity of CAD.

\footnotetext{
*Correspondence to: Dr. Durmuş Yıldıray Şahin, Department of Cardiology, Adana Numune Training and Research Hospital, Adana 01170, Turkey. E-mail: dysahin79@hotmail.com
}

Received 16 February 2013; Accepted 3 June 2013

DOI $10.1002 /$ jcla.21643

Published online in Wiley Online Library (wileyonlinelibrary.com). 


\section{METHODS}

\section{Study Population}

Between May 2012 and November 2012, 209 consecutive patients (109 males and 100 females; mean age: $63.1 \pm 10.0$ years) with angiographically proven CAD and 102 angiographically normal control subjects (62 males and 40 females; mean age; $61.3 \pm 13.7$ years) were included to the study. Angiography was performed for the investigation of ischemic heart disease based on clinical indications (typically chest discomfort and/or abnormal stress test results). The patients with coronary lesions with a diameter stenosis $\geq 50 \%$ in vessels $\geq 1.5 \mathrm{~mm}$ were included in the study. All patients were clinically stable. Exclusion criteria were the presence of neoplastic disease, heart failure, recent major surgical procedure, evidence of hypercalcaemia, and systemic inflammatory conditions, such as infection, liver, or kidney disease. Patients with previous and new onset myocardial infarction and angina episodes in last $48 \mathrm{hr}$ before hospitalization, who had undergone coronary angioplasty or bypass surgery, and those with valvular, myocardial, or pericardial disease were also excluded from the study. None of the patients and control subjects was taking calcium or vitamin D supplements. The study was conducted according to the recommendations set forth by the Declaration of Helsinki on biomedical research involving human subjects. The Institutional Ethics Committee approved the study protocol, and each participant provided written, informed consent.

A detailed medical history was taken and a complete physical examination was conducted in all subjects. Histories of diabetes, hypertension, hyperlipidemia, smoking, and family history were recorded for all participants. In addition, systolic blood pressure (SBP) and diastolic blood pressure (DBP) were recorded. Body mass index (BMI) was computed as weight divided by height squared $\left(\mathrm{kg} / \mathrm{m}^{2}\right)$.

Standard two-dimensional examinations were performed using commercially available equipment (Vivid7, GE Vingmed Sound, Horten, Norway) with a 2.53.5 MHz transducer before angiography. Ejection fraction was determined by using Simpson's method, according to the suggestions of the American Society of Echocardiography (14).

\section{Blood Samples}

Fasting venous blood samples were obtained from all patients to determine laboratory parameters. Glucose, creatinine, and lipid profiles for blood samples were analyzed for each patient. High-sensitivity $\mathrm{C}$ reactive protein (hs-CRP) was measured using BN2 model nephelometer.

Serum calcium (8.2-10.2 mg/dl) was measured using a Cobas c501 Analyzer (Roche Diagnostics, Tokyo, Japan).
Parathyroid hormone was measured using an electrochemiluminescence immunoassay on the Cobas e601 Analyzer (Roche Diagnostics). The intra-assay coefficient of variation $(\mathrm{CV})$ was $2.70 \%$ and interassay $\mathrm{CV}$ was $5.23 \%$.

Serum vitamin D level was measured using a direct competitive chemiluminescent immunoassay (Elecsys; Roche Diagnostics, Mannheim, Germany). We also recorded dates of 25-hyroxyvitamin D measurement and categorized them into two seasons: summer (May-August) and fall (September-November). Serum 25(OH)D level below $20 \mathrm{ng} / \mathrm{ml}$ was defined as vitamin D deficiency as in previous studies $(1,15)$. Intra- and interassay CVs were below $4.3 \%$ and $7.2 \%$, respectively.

\section{Coronary Angiography and SYNTAX Score}

All participants underwent coronary angiography with the Judkins technique. SYNTAX score and angiographic analysis coronary lesions leading to $\geq 50 \%$ diameter stenosis in vessels $\geq 1.5 \mathrm{~mm}$ were scored separately and added together to provide the cumulative SYNTAX score that was prospectively calculated using the SYNTAX score algorithm on the baseline diagnostic angiogram (10). Two experienced interventional cardiologists analyzed the SYNTAX score; the opinion of a third analyst was obtained and the final judgment was made by consensus in case of disagreement. The final score was calculated from the individual lesion scores by analysts who were blinded to procedural data and clinical outcome.

\section{Statistical Analysis}

All analyses were conducted using SPSS 17.0 (SPSS for Windows 17.0, Chicago, IL). Comparison of categorical variables between the groups was performed using the Chi-square test. The Kolmogorov-Smirnov test was performed to evaluate the normality of distribution of all continuous variables. hs-CRP value was normalized by logarithmic transformation and the values were used for statistical analysis. Comparisons of continuous variables between the two groups were performed using the independent samples $t$-test. The correlation between vitamin D and continuous parameters was assessed by the Pearson correlation analysis, whereas the correlation between vitamin $\mathrm{D}$ and nominal variables, such as gender, HT, and diabetes, was evaluated with point biserial correlation analysis. A multivariate stepwise linear regression analysis was performed to identify the independent associations of vitamin D. Staged multivariate regression models were constructed for the current analysis. Model 1: standard model, all significant variables on bivariate analysis (age, gender, SYNTAX score, BMI, low-density lipoprotein cholesterol (LDL-C), hs-CRP, diabetes and hypertension) were entered into model. Model 2: adjusted 
as model 1 plus parathyroid hormone. A multivariate logistic regression analysis was performed to identify the independent predictors of CAD. A two-tailed $P<0.05$ was considered statistically significant.

\section{RESULTS}

Deficiency of vitamin D levels was observed in $95.7 \%$ of patients with CAD and $80.2 \%$ of controls in this study. The concentration of vitamin D was $9.4 \pm 5.1 \mathrm{ng} / \mathrm{ml}$ in patients. The vitamin $\mathrm{D}$ concentrations measured in fall were similar to that in summer $(9.2 \pm 5.6 \mathrm{ng} / \mathrm{ml}$ vs. $9.7 \pm$ $6.4 \mathrm{ng} / \mathrm{ml}, P=0.332$ ) in patients.

Comparisons of baseline, clinical, laboratory, and echocardiographic characteristics of subjects were shown in Table 1. The patients group had higher frequencies of diabetes, hypertension, smoking, hyperlipidemia, family history, higher level of SBP, fasting glucose, hs-CRP, and creatinine and lower level of vitamin $\mathrm{D}$ and high-density lipoprotein cholesterol than the control group $(P<0.05$, for all). Furthermore, frequencies of acetyl salicylic acid use, angiotensin converting enzyme inhibitor use, beta blocker and statin use were higher in patient group compared with control group $(P<0.05$, for all). Calcium levels and parathyroid hormone values of groups were similar $(P>0.05)$. When patient group was divided into two groups according to the median SYNTAX score (Table 2), level of vitamin D was significantly lower in patients with high SYNTAX score $(\geq 16)$ than patients with low SYNTAX score $(<16 ; P<0.05)$. However, previous statin use was similar between groups.

Bivariate and multivariate relationships of vitamin D in patient group were summarized in Tables 3 and 4. Vitamin D level was significantly associated with age $(r=-0.323$, $P<0.001)$, gender $(r=-0.192, P=0.0054)$, SYNTAX score $(r=-0.549, P<0.001)$, BMI $(r=-0.174, P=$ $0.012)$, LDL-C $(r=-0.148, P=0.033)$, hs-CRP $(r=$ $-0.230, P=0.001)$, diabetes $(r=-0.320, P<0.001)$, and hypertension $(r=-0.380, P<0.001)$ in bivariate analysis. Relationship between SYNTAX score and vitamin D level was demonstrated in Figure 1.

Multivariate regression analysis showed that serum 25hydroxyvitamin D level was independently associated with SYNTAX score $(\beta=-0,396, P<0,001)$, hypertension $(\beta=-0,183, P=0,003)$, hs-CRP $(\beta=-0,141$, $P=0,014)$, and BMI $(\beta=-0,135, P=0,023)$ in model 1 and remained significant with additional adjustment for parathyroid hormone.

Hypertension ( $\beta=0.541,95 \%$ CI: $0.303-0.967, P=$ $0.038)$, diabetes $(\beta=0.966,95 \%$ CI: 0.941-0.991, $P=$ $0.007)$, creatinine level $(\beta=0.534,95 \%$ CI: $0.308-0.928$, $P=0.025)$, and vitamin D level $(\beta=0.933,95 \% \mathrm{CI}: 892$
975, $P=0.002$ ) were independent predictors of CAD on multivariate logistic regression analysis.

\section{DISCUSSION}

The main findings of this study are (1) vitamin D level is lower in patient group compared with control group; (2) the vitamin D level is independently associated with SYNTAX score as well as hypertension, BMI, and hs-CRPadjustment for parathyroid hormone did not modify these associations; (3) hypertension, diabetes, creatinine level, and vitamin D level were independent predictors of CAD on multivariate logistic regression analysis.

Our results of an extraordinarily high prevalence of vitamin D deficiency $(96 \%)$ in the patients admitted for stable CAD are consistent with data associating cardiovascular disease with vitamin D deficiency (9). Several large cross-sectional and longitudinal observational studies have shown that low levels of vitamin $\mathrm{D}$ are associated with an increased risk of CAD $(6,7)$. It was shown that levels of vitamin $\mathrm{D}<30 \mathrm{ng} / \mathrm{ml}$ were associated with a greater risk of incident myocardial infarction even after adjustment for risk factors known to be associated with CAD (16). Moreover, low vitamin D level has emerged as a key biologic predictor of increased rates of cardiovascular disease risk factors, such as hypertension $(17,18)$, obesity (19), diabetes mellitus (20), and the metabolic syndrome (21) and progression factors (e.g., inflammation; (8)).

The present study shows that vitamin D level is inversely associated with extent and complexity of CAD assessed with SYNTAX score. Relationship between low vitamin $\mathrm{D}$ level and CAD is well known. However, relationship between vitamin $\mathrm{D}$ level and severity of CAD was investigated in only one study (22). In that study, severity of CAD was assessed by using the Gensini score. Nevertheless, Gensini score does not reflect the exact complexity of CAD. The factors related with complex lesions, such as bifurcation, calcification, lesion length, and tortuosity are not considered in Gensini score. SYNTAX score is a novel method and quantifies extent and complexity of CAD $(10,23)$. Also, SYNTAX score predicts morbidity and mortality in patients with CAD $(11,12)$. Calcification is the common pathognomonic change of nearly all angiographically significant lesions (24). Previous studies have demonstrated that lower serum vitamin D levels are associated with an increased risk for coronary calcification (25). Increased risk for developing new-onset coronary calcification during 3 years of follow-up was reported in the Multi-Ethnic Study of Atherosclerosis (26). In the present study, relationship between coronary calcification and serum vitamin D level was not investigated. However, SYNTAX scoring method incorporates coronary calcification. The significant association between vitamin 
TABLE 1. Comparison of Baseline, Clinical, Laboratory, and Echocardiographic Characteristics of Groups

\begin{tabular}{|c|c|c|c|}
\hline Variables & Control group $(n=102)$ & Patient group $(n=209)$ & $P$ value \\
\hline Age (year) & $61.3 \pm 13.7$ & $63.1 \pm 10.0$ & 0.191 \\
\hline Gender $(\text { male })^{\mathrm{a}}$ & $62(60.8 \%)$ & $109(52.3 \%)$ & 0.094 \\
\hline Diabetes mellitus, $n(\%)^{\mathrm{a}}$ & $18(17.6 \%)$ & $75(35.9 \%)$ & 0.001- \\
\hline Hypertension, $n(\%)^{\mathrm{a}}$ & $34(33.3 \%)$ & $119(56.9 \%)$ & $<0.001$ \\
\hline Smoking, $n(\%)^{\mathrm{a}}$ & $24(23.5 \%)$ & $74(35.5 \%)$ & 0.022 \\
\hline Hyperlipidemia, $n(\%)^{\mathrm{a}}$ & $27(26.5 \%)$ & $76(36.9 \%)$ & 0.044 \\
\hline Family history, $n(\%)^{\mathrm{a}}$ & $38(37.3 \%)$ & $116(55.5 \%)$ & 0.002 \\
\hline $\operatorname{BMI}\left(\mathrm{kg} / \mathrm{m}^{2}\right)$ & $29.0 \pm 5.0$ & $28.5 \pm 4.9$ & 0.374 \\
\hline $\mathrm{SBP}(\mathrm{mmHg})$ & $122.2 \pm 13.1$ & $128.2 \pm 14.9$ & 0.001 \\
\hline $\mathrm{DBP}(\mathrm{mmHg})$ & $77.7 \pm 9.6$ & $78.3 \pm 8.4$ & 0.579 \\
\hline Heart rate (beat/min) & $75.2 \pm 12.4$ & $76.3 \pm 13.9$ & 0.517 \\
\hline Fasting glucose $(\mathrm{mg} / \mathrm{dl})$ & $109,1 \pm 47,9$ & $139,5 \pm 89,1$ & $<0.001$ \\
\hline $\mathrm{TC}(\mathrm{mg} / \mathrm{dl})$ & $195.6 \pm 41.4$ & $198.9 \pm 43.4$ & 0.618 \\
\hline Triglyceride (mg/dl) & $154.8 \pm 80.6$ & $176.0 \pm 130.4$ & 0.133 \\
\hline HDL-C (mg/dl) & $45.1 \pm 11.5$ & $41.4 \pm 10.6$ & 0.005 \\
\hline LDL-C (mg/dl) & $119.5 \pm 33.3$ & $122.3 \pm 36.4$ & 0.516 \\
\hline hs-CRP (mg/dl) & & & 0.005 \\
\hline median (25th-75th) & $0.30(0.10-0.70)$ & $0.40(0.20-1.20)$ & \\
\hline Creatinine (mg/dl) & $0.72 \pm 0.15$ & $0.80 \pm 0.20$ & $<0.001$ \\
\hline Vitamin D (ng/ml) & $13.1 \pm 8.9$ & $9.4 \pm 5.0$ & $<0.001$ \\
\hline Calcium (mg/dl) & $9.2 \pm 0.6$ & $9.1 \pm 0.5$ & 0.550 \\
\hline Parathyroid hormone $(\mathrm{pg} / \mathrm{ml})$ & $46.4 \pm 20.0$ & $47.3 \pm 24.7$ & 0.771 \\
\hline Ejection fraction $(\%)$ & $60.0 \pm 8.1$ & $58.0 \pm 6.7$ & 0.070 \\
\hline ASA, $n(\%)^{\mathrm{a}}$ & $17(16.6 \%)$ & $88(42.1 \%)$ & $<0.001$ \\
\hline ACE inhibitor, $n(\%)^{\mathrm{a}}$ & $18(17.6 \%)$ & $69(33 \%)$ & 0.003 \\
\hline ARB use, $n(\%)^{\mathrm{a}}$ & $4(3 \%)$ & $15(7 \%)$ & 0.190 \\
\hline Beta blocker use n $(\%)^{\mathrm{a}}$ & $12(11.7 \%)$ & $76(36.3 \%)$ & $<0.001$ \\
\hline Statin use, $n(\%)^{\mathrm{a}}$ & $8(7.8 \%)$ & $30(14.3 \%)$ & 0.007 \\
\hline Nitrate use, $n(\%)^{\mathrm{a}}$ & $2(1.9 \%)$ & $8(3.8 \%)$ & 0.490 \\
\hline
\end{tabular}

BMI, body mass index; SBP, systolic blood pressure; DBP, diastolic blood pressure; TC, total cholesterol; HDL-C, high-density lipoprotein cholesterol; LDL-C, low-density lipoprotein cholesterol; hs-CRP, high-sensitive C reactive protein; ASA, acetyl salicylic acid; ACE, angiotensin converting enzyme; ARB, angiotensin receptor blocker.

${ }^{\mathrm{a}}$ Chi-square.

Significance $p$ values $(p<0.05)$ were indicated in boldface.

D deficiency and CAD as evaluated by coronary CT angiography was reported in a previous study (27). In another study, it was reported that there was an interaction between vitamin $\mathrm{D}$ levels and presence of angiographic severe stenosis (28). On the other hand, previous one study did not find an association between different vitamin D levels and prevalent angiographic CAD (29).

The pathophysiological mechanism behind the relation between extent and complexity of CAD and lower serum vitamin D levels is not well defined. Several mechanisms may be suggested for the relationship between extent and complexity of CAD and vitamin D levels. Vitamin D may play a role in the pathogenesis of cardiovascular diseasethrough a direct involvement in the process of plaque formation and progression $(8,13)$. Proliferation of vascular smooth muscle cells is a key event during plaque formation in atherosclerotic heart disease (30). Vitamin $\mathrm{D}$ modulates key process involved in the pathogenesis of cardiovascular disease including vascular smooth muscle cell proliferation (31). In addition, it was shown that

TABLE 2. Vitamin D, Calcium, and Parathyroid Hormone Levels According to Median Syntax Score in Patient Group

\begin{tabular}{lcc}
\hline Variables & Low SYNTAX score $(<16.0)$ & High SYNTAX score $(\geq 16)$ \\
\hline Vitamin D $(\mathrm{ng} / \mathrm{ml})$ & $11.5 \pm 4.8$ & $7.4 \pm 4.3$ \\
Calcium $(\mathrm{mg} / \mathrm{dl})$ & $9.2 \pm 0.47$ & $\mathbf{< 0 . 0 0 1} \pm 0.57$ \\
Parathyroid hormone $(\mathrm{pg} / \mathrm{ml})$ & $46.5 \pm 25.9$ & 0.153 \\
Statin use, $n(\%)$ & $13(12.5)$ & 0.645 \\
\hline
\end{tabular}

Significance $p$ value $(p<0.05)$ was indicated in boldface. 
TABLE 3. Bivariate Relationship of Vitamin D in Patient Group

\begin{tabular}{|c|c|c|}
\hline Variables & $\begin{array}{c}\text { Pearson correlation } \\
\text { coefficient }\end{array}$ & $P$ value \\
\hline Age $(\text { year })^{\mathrm{a}}$ & -0.323 & $<0.001$ \\
\hline Gender $^{\mathrm{b}}$ & $-0,192$ & 0.0054 \\
\hline SYNTAX score ${ }^{\mathrm{a}}$ & -0.549 & $<0.001$ \\
\hline Body mass index $\left(\mathrm{kg} / \mathrm{m}^{2}\right)^{\mathrm{a}}$ & -0.174 & 0.012 \\
\hline LDL-C $(\mathrm{mg} / \mathrm{dl})^{\mathrm{a}}$ & -0.148 & 0.033 \\
\hline hs-CRP $(\mathrm{mg} / \mathrm{dl})^{\mathrm{a}}$ & -0.230 & 0.001 \\
\hline Diabetes, $n(\%)^{\mathrm{b}}$ & -0.320 & $<0.001$ \\
\hline Hypertension, $n(\%)^{\mathrm{b}}$ & -0.380 & $<0.001$ \\
\hline
\end{tabular}

LDL-C, low-density lipoprotein cholesterol; hs-CRP, high-sensitive C reactive protein.

${ }^{a}$ From Pearson correlation analysis.

${ }^{b}$ From point biserial correlation analysis.

TABLE 4. Multivariate Relationship of Vitamin D in Patient Group

\begin{tabular}{lccccc}
\hline & \multicolumn{2}{c}{ Model 1} & & \multicolumn{2}{c}{ Model 2 } \\
\cline { 2 - 3 } \cline { 6 - 7 } Variables & $\beta$ & $P$ & & $\beta$ & $P$ \\
\hline Age (year) & -0.121 & 0.055 & & -0.105 & 0.075 \\
Gender & -0.105 & 0.1 & & -0.112 & 0.09 \\
SYNTAX score & -0.396 & $<\mathbf{0 . 0 0 1}$ & & -0.426 & $<\mathbf{0 . 0 0 1}$ \\
Body mass index $\left(\mathrm{kg} / \mathrm{m}^{2}\right)$ & -0.135 & $\mathbf{0 . 0 2 3}$ & & -0.145 & $\mathbf{0 . 0 0 1}$ \\
LDL-C $(\mathrm{mg} / \mathrm{dl})$ & -0.071 & 0.230 & & -0.097 & 0.152 \\
hs-CRP $(\mathrm{mg} / \mathrm{dl})$ & -0.141 & $\mathbf{0 . 0 1 4}$ & -0.138 & $\mathbf{0 . 0 1 0}$ \\
Diabetes, $n(\%)$ & -0.023 & 0.721 & -0.027 & 0.668 \\
Hypertension, $n(\%)$ & -0.183 & $\mathbf{0 . 0 0 3}$ & -0.194 & $\mathbf{0 . 0 0 1}$ \\
\hline
\end{tabular}

Model 1 (standard model): all significant variables (age, gender, SYNTAX score, body mass index, LDL-C, hs-CRP, diabetes, and hypertension) were entered into model.

Model 2: adjusted as in model 1 plus parathyroid hormone level.

LDL-C, low-density lipoprotein cholesterol; hs-CRP, high-sensitive C reactive protein; $\beta$, standardized $\beta$-regression coefficient.

Significance $p$ values $(p<0.05)$ were indicated in boldface.

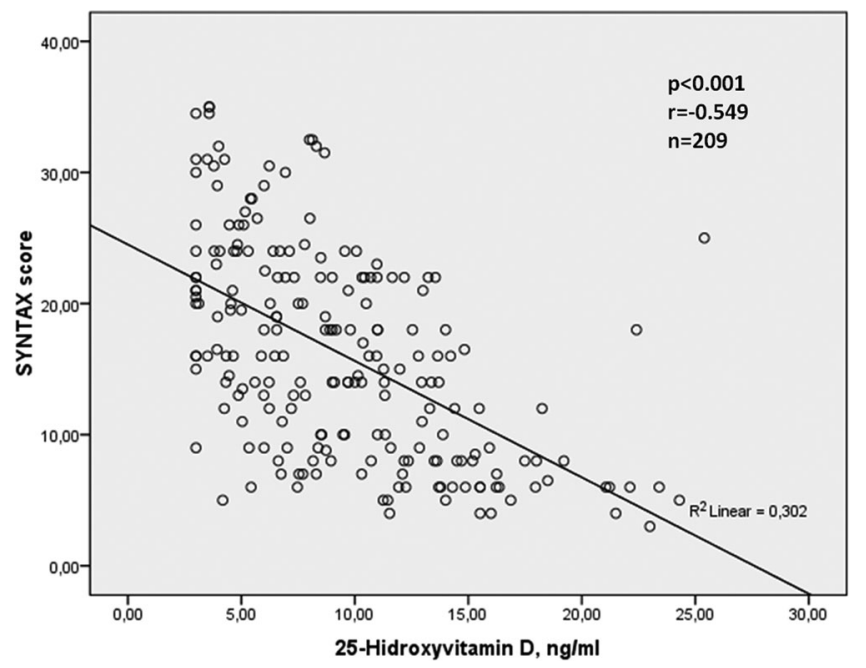

Fig. 1. Scatter graph showing strong correlation between SYNTAX score and 25-hydroxyvitamin D. active vitamin D suppresses foam-cell formation by reducing macrophage cholesterol uptake in diabetics (32). Lower serum vitamin D levels by increasing macrophage cholesterol uptake may facilitate coronary atherosclerosis. Inflammation is a key factor driving the processes of plaque formation, progression, and rupture in patients with atherosclerotic CAD (33). Vitamin D deficiency promotes stimulation of systemic and vascular inflammation (34). Recent studies have shown an inverse relationship between vitamin D levels and inflammation (8). The present study shows that there is independent inverse relationship between vitamin D and hs-CRP. The relationship between lower serum vitamin $\mathrm{D}$ level and increased inflammation may mediate to more complex lesions in stable CAD. Also, the downregulation of vitamin $\mathrm{D}$ binding protein was confirmed and marked in multivessel CAD patients (35). Moreover, in a previous experimental study, lower concentrations of vitamin $\mathrm{D}$ receptors in a main coronary artery were associated with greater atherosclerotic plaque size in postmenopausal female monkeys (36). This experimental study may contribute to relationship between lower vitamin D level and extent and complexity of CAD. Vitamin D may also modulate key processes involved in the pathogenesis of cardiovascular disease, including platelet aggregation/thrombogenesis (37), and the renin-angiotensin system (38).

In the current study, lower vitamin D level is independently associated with the diagnosis of hypertension. Vitamin D suppresses renin-angiotensin system, and this condition is effective on blood pressure (38). Previous studies reported that low serum vitamin $\mathrm{D}$ level was associated with a higher prevalence of hypertension $(17,18)$.

Several studies have reported on the association between obesity and low vitamin D levels (19). Patients with $25(\mathrm{OH}) \mathrm{D}$ deficiency had significantly higher BMIs compared to those with sufficient levels of $25(\mathrm{OH}) \mathrm{D}$ (39). This result supports research that suggests sequestering of $25(\mathrm{OH}) \mathrm{D}$ in adipose tissue and decreasing the amount of circulating $25(\mathrm{OH}) \mathrm{D}(39)$. In the present study, lower vitamin D level is independently associated with BMI as well as SYNTAX score.

\section{Study Limitations}

The present study has some significant limitations: First, $80.2 \%$ of control group had vitamin D deficiency in the present study. However, control group did not consist of completely healthy individuals. In the present study, subjects with normal coronary artery were selected as control group. It has been known that serum vitamin D levels vary with region, seasonality, and altitude due to possible effect of sunlight. But, we had no data on sun exposure time. However, the half-life of vitamin D is approximately 3 weeks, but serum vitamin D levels 
may change throughout the day and season of the year. So, a single measurement may not reflect actual vitamin D status and coronary atherosclerosis progresses over many years. Fourth, previous medications, particularly statin use (40), may affect the vitamin D levels. However, none of the medications was associated with vitamin $\mathrm{D}$ level in both bivariate and multivariate analysis. Finally, 1,25-dihydroxyvitamin $\mathrm{D}_{3}$ is the active metabolite. 1,25-Dihydroxyvitamin $\mathrm{D}_{3}$ is not measured in the present study. 25-Hydroxyvitamin D has important autocrine and paracrine roles in the synthesis and determination of intracellular levels of 1,25-dihydroxyvitamin $\mathrm{D}_{3}$ (41). $25-$ Hydroxyvitamin $\mathrm{D}$ is the only vitamin $\mathrm{D}$ metabolite that is used to determine vitamin D insufficiency and deficiency and 25-hydroxyvitamin D levels might not reflect circulating 1,25-dihydroxyvitamin $\mathrm{D}_{3}$ levels.

\section{CONCLUSIONS}

The present study shows that vitamin D level of patient group is lower compared with control group. Vitamin D level is independently associated with extent and complexity of CAD assessed with SYNTAX score as well as hs-CRP, BMI, and hypertension. Hypertension, diabetes, creatinine level, and vitamin D level were independent predictors of CAD. Lower vitamin D level may play a role on presence and severity of CAD.

\section{CONFLICTS OF INTERESTS}

The authors report no conflicts of interest.

\section{REFERENCES}

1. Lee JH, Gadi R, Spertus JA, Tang F, O'Keefe JH. Prevalence of vitamin $\mathrm{D}$ deficiency in patients with acute myocardial infarction. Am J Cardiol 2011;107:163-168.

2. Goswami R, Gupta N, Goswami D, Marwaha RK, Tandon N, Kochupillai N. Prevalence and significance of low 25-hydroxyvitamin D concentrations in healthy subjects in Delhi. Am J Clin Nutr 2000;72:472-475.

3. Reis JP, von Mühlen D, Michos ED, et al. Serum vitamin $\mathrm{D}$, parathyroid hormone levels, and carotid atherosclerosis. Atherosclerosis 2009;207:585-590.

4. Lavie CJ, Lee JH, MIlani RV. Vitamin D and cardiovascular disease. J Am Coll Cardiol 2011;5:1547-1556.

5. Anderson JL, May HT, Horne BD, et al. Relation of vitamin D deficiency to cardiovascular risk factors, disease status and incident events in a general healthcare population. Am J Cardiol 2010;106:963-968.

6. Giovannucci E, Liu Y, Hollis BW, Rimm EB. 25-hydroxyvitamin $\mathrm{D}$ and risk of myocardial infarction in men: A prospective study. Arch Intern Med 2008;168:1174-1180.

7. Wang TJ, Pencina MJ, Booth SL, et al. Vitamin D deficiency and risk of cardiovascular disease. Circulation 2008;11:503511.

8. Dobnig H, Pilz S, Scharnagl H, et al. Independent association of low serum 25-hydroxyvitamin D and 1,25- dihydroxyvitamin D levels with all-cause and cardiovascular mortality. Arch Intern Med 2008;168:1340-1349.

9. Vacek JL, Vanga SR, Good M, Lai SM, Lakkireddy D, Howard PA. Vitamin D deficiency and supplementation and relation to cardiovascular health. Am J Cardiol 2012;109:359-363.

10. Sianos G, Morel MA, Kappetein AP, et al. The SYNTAX score: An angiographic tool grading the complexity of coronary artery disease. EuroIntervention 2005;1:219-227.

11. Capodanno D, Di Salvo ME, Cincotta G, Miano M, Tamburino C, Tamburino C. Usefulness of the SYNTAX score for predicting clinical outcome after percutaneous coronary intervention of unprotected left main coronary artery disease. Circ Cardiovasc Interv 2009;2:302-308.

12. Capodanno D, Capranzano P, Di Salvo ME, et al. Usefulness of SYNTAX score to select patients with left main coronary artery disease to be treated with coronary artery bypass graft. J Am Coll Cardiol Intv 2009;2:731-738.

13. Tarcin O, Yavuz DG, Ozben B, et al. Effect of vitamin D deficiency and replacement on endothelial function in asymptomatic subjects. J Clin Endocrinol Metab 2009;94:4023-4030.

14. Schiller NB, Shah PM, Crawford M, et al. Recommendations for quantitation of the left ventricle by two-dimensional echocardiography. American Society of Echocardiography Committee on Standards, Subcommittee on Quantitation of Two-Dimensional Echocardiograms. J Am Soc Echocardiogr 1989;2:358-367.

15. Lee JH, O'Keefe JH, Bell D, et al. Vitamin D deficiency an important, common, and easily treatable cardiovascular risk factor? J Am Coll Cardiol 2008;52:1949-1956.

16. Giovannucci E, Liu Y, Hollis BW, Rimm EB. 25-hydroxyvitamin $\mathrm{D}$ and risk of myocardial infarction in men: A prospective study. Arch Intern Med 2008;168:1174-1180.

17. Scragg R, Sowers M, Bell C. Serum 25-hydroxyvitamin D, ethnicity, and blood pressure in the Third National Health and Nutrition Examination Survey. Am J Hypertens 2007;20:713-719.

18. Snijder MB, Lips P, Seidell JC, et al. Vitamin D status and parathyroid hormone levels in relation to blood pressure: A populationbased study in older men and women. J Intern Med 2007;261:558565.

19. Lee P, Greenfield JR, Seibel MJ, Eisman JA, Center JR. Adequacy of vitamin D supplementation in severe deficiency is dependent on body mass index. Am J Med 2009;122:1056-1060.

20. Scragg R, Sowers M, Bell C. Serum 25-hydroxyvitamin D, diabetes, and ethnicity in the Third National Health and Nutrition Examination Survey. Diabetes Care 2004;27:2813-2818.

21. Thomas GN, ó Hartaigh B, Bosch JA, et al. Vitamin D levels predict all-cause and cardiovascular disease mortality in subjects with the metabolic syndrome: The Ludwigshafen Risk and Cardiovascular Health (LURIC) Study. Diabetes Care 2012;35:1158-1164.

22. Akin F, Ayça B, Köse N, et al. Serum vitamin D levels are independently associated with severity of coronary artery disease. J Investig Med 2012;60:869-873.

23. Aksakal E, Tanboga IH, Kurt M, et al. Predictors of coronary lesions complexity in patients with stable coronary artery disease. Angiology 2013;64:304-309.

24. Honye J, Mahon DJ, Jain A, et al. Morphological effects of coronary balloon angioplasty in vivo assessed by intravascular ultrasound imaging. Circulation 1992;85:1012-1025.

25. Watson KE, Abrolat ML, Malone LL, et al. Active serum vitamin D levels are inversely correlated with coronary calcification. Circulation 1997;96:1755-1760.

26. de Boer IH, Kestenbaum B, Shoben AB, Michos ED, Sarnak MJ, Siscovick DS. 25 Hydroxyvitamin D levels inversely associate with risk for developing coronary artery calcification. J Am Soc Nephrol 2009;20:1805-1812. 


\section{8 Şeker et al.}

27. Lim S, Shin H, Kim MJ. Vitamin D inadequacy is associated with significant coronary artery stenosis in a community-based elderly cohort: The Korean Longitudinal Study on Health and Aging. J Clin Endocrinol Metab 2011;97:169-178.

28. Shor R, Tirosh A, Shemesh L, et al. 25 Hydroxyvitamin D levels in patients undergoing coronary artery catheterization. Eur J Intern Med 2012;23:470-473.

29. Pilz S, März W, Wellnitz B, et al. Association of vitamin D deficiency with heart failure and sudden cardiac death in a large crosssectional study of patients referred for coronary angiography. J Clin Endocrinol Metab 2008;93:3927-3935.

30. Zhang C. The role of inflammatory cytokines in endothelial dysfunction. Basic Res Cardiol 2008;103:398-406.

31. Mitsuhashi T, Morris RC, Jr, Ives HE. 1,25-Dihydroxyvitamin D3 modulates growth of vascular smooth muscle cells. J Clin Invest 1991;87:1889-1895.

32. Oh J, Weng S, Felton SK, et al. 1,25(OH)2 vitamin D inhibits foam cell formation and suppresses macrophage cholesterol uptake in patients with type 2 diabetes mellitus. Circulation 2009;120:687698.

33. Libby P. Vascular biology of atherosclerosis: Overview and state of the art. Am J Card 2003;91:3A-6A.

34. van Etten E, Mathieu C. Immunoregulation by 1,25dihydroxyvitamin D3: Basic concepts. J Steroid Biochem Mol Biol 2005;97:93-101.
35. Rocchiccioli S, Andreassi MG, Cecchettini A, Carpeggiani C, L'Abbate A, Citti L. Correlation between vitamin D binding protein expression and angiographic-proven coronary artery disease. Coron Artery Dis 2012;23:426-431.

36. Schnatz PF, Nudy M, O'Sullivan DM, et al. The quantification of vitamin $\mathrm{D}$ receptors in coronary arteries and their association with atherosclerosis. Maturitas 2012;73:143147.

37. Aihara K, Azuma H, Akaike M, et al. Disruption of nuclear vitamin $\mathrm{D}$ receptor gene causes enhanced thrombogenicity in mice. J Biol Chem 2004;279:35798-35802.

38. Li YC. Vitamin D regulation of the renin-angiotensin system. J Cell Biochem 2003;88:327-331.

39. Lin E, Armstrong-Moore D, Liang Z, et al. Contribution of adipose tissue to plasma 25-hydroxyvitamin D concentrations during weight loss following gastric bypass surgery. Obesity (Silver Spring) 2011;19:588-594.

40. Bittner V, Wenger NK, Waters DD, DeMicco DA, Messig M, LaRosa JC. Vitamin D levels do not predict cardiovascular events in statin-treated patients with stable coronary disease. Am Heart J 2012;164:387-393.

41. Oudshoorn C, van der Cammen TJ, McMurdo ME, van Leeuwen JP, Colin EM. Ageing and vitamin D deficiency: Effects on calcium homeostasis and considerations for vitamin D supplementation. $\mathrm{Br}$ J Nut 2009;101:1597-1606. 